\title{
On the existence of mild solutions for nonlocal differential equations of the second order with conformable fractional derivative
}

\author{
Mustapha Atraoui $^{1 *}$ and Mohamed Bouaouid ${ }^{2}$
}

\section{"Correspondence:}

m.atraoui19@gmail.com

'Department of Mathematics,

Faculty of Applied Sciences, Ait

Melloul Campus, Ibn Zohr

University, BP 6146, 86150, Agadir,

Morocco

Full list of author information is

available at the end of the article

\section{Springer}

\begin{abstract}
In the work (Bouaouid et al. in Adv. Differ. Equ. 2019:21, 2019), the authors have used the Krasnoselskii fixed point theorem for showing the existence of mild solutions of an abstract class of conformable fractional differential equations of the form: $\frac{d^{\alpha}}{d t^{\alpha}}\left[\frac{d^{\alpha} x(t)}{d t^{\alpha}}\right]=A x(t)+f(t, x(t)), t \in[0, \tau]$ subject to the nonlocal conditions $x(0)=x_{0}+g(x)$ and $\frac{d^{\alpha} x(0)}{d t^{\alpha}}=x_{1}+h(x)$, where $\frac{d^{\alpha}(\cdot)}{d t^{\alpha}}$ is the conformable fractional derivative of order $\alpha \in] 0,1]$ and $A$ is the infinitesimal generator of a cosine family $(\{C(t), S(t)\})_{t \in \mathbb{R}}$ on a Banach space $X$. The elements $x_{0}$ and $x_{1}$ are two fixed vectors in $X$, and $f, g, h$ are given functions. The present paper is a continuation of the work (Bouaouid et al. in Adv. Differ. Equ. 2019:21, 2019) in order to use the Darbo-Sadovskii fixed point theorem for proving the same existence result given in (Bouaouid et al. in Adv. Differ. Equ. 2019:21, 2019) [Theorem 3.1] without assuming the compactness of the family $(S(t))_{t>0}$ and any Lipschitz conditions on the functions $g$ and $h$.
\end{abstract}

MSC: 34A08; 47D09

Keywords: Fractional differential equations; Cosine family of linear operators; Conformable fractional derivative; Nonlocal conditions; Measure of noncompactness

\section{Introduction}

Classical derivatives appear in several mathematical models in various areas of science such as physics, engineering, biology, finance, and so on. However, there are many phenomena that may not depend only on the time moment but also on the former time history, which cannot be modeled utilizing the classical derivatives. For this reason, many authors try to replace the classical derivatives with the so-called fractional derivatives in numerous contributions [2-17], because it has been proven that this last kind of derivatives is a very good way to describe processes with memory. According to the literature of fractional calculus, it is remarkable that there are many approaches to defining fractional derivatives, and each definition has advantages compared to others [18-22]. In consequence, many researchers have paid attention to propose new fractional derivatives in order to deal better with modeling of evolutionary phenomena [23, 24]. In the work [23], the authors proposed the so-called fractional conformable derivative, which quickly became the subject of many

(c) The Author(s) 2021. This article is licensed under a Creative Commons Attribution 4.0 International License, which permits use sharing, adaptation, distribution and reproduction in any medium or format, as long as you give appropriate credit to the original author(s) and the source, provide a link to the Creative Commons licence, and indicate if changes were made. The images or other third party material in this article are included in the article's Creative Commons licence, unless indicated otherwise in a credit line to the material. If material is not included in the article's Creative Commons licence and your intended use is not permitted by statutory regulation or exceeds the permitted use, you will need to obtain permission directly from the copyright holder. To view a copy of this licence, visit http://creativecommons.org/licenses/by/4.0/. 
research papers [1, 25-54]. For example, in [1] the authors proved the existence of mild solutions for the following nonlocal conformable fractional Cauchy problem:

$$
\left\{\begin{array}{l}
\frac{d^{\alpha}}{d t^{\alpha}}\left[\frac{d^{\alpha} x(t)}{d t^{\alpha}}\right]=A x(t)+f(t, x(t)), \quad t \in[0, \tau], \\
x(0)=x_{0}+g(x), \\
\frac{d^{\alpha} x(0)}{d t^{\alpha}}=x_{1}+h(x),
\end{array}\right.
$$

which is a natural extension of the works $[55,56]$ in the frame of the conformable fractional derivative, where $A$ is the infinitesimal generator of a cosine family $\{C(t), S(t)\}_{t \in \mathbb{R}}$ on a Banach space $(X,\|\cdot\|)$ and $\frac{d^{\alpha}(\cdot)}{d t^{\alpha}}$ presents the conformable fractional derivative of order $\alpha \in] 0,1]$. The elements $x_{0}$ and $x_{1}$ are two fixed vectors in $X$, and $f:[0, \tau] \times X \longrightarrow X, g$ : $\mathcal{C} \longrightarrow X, h: \mathcal{C} \longrightarrow X$ are given functions, where $\left(\mathcal{C},|\cdot|_{c}\right)$ is the space of continuous functions from $[0, \tau]$ into $X$ with $|\cdot|_{c}$ being the uniform norm topology in $\mathcal{C}$. The expressions $x(0)=$ $x_{0}+g(x)$ and $\frac{d^{\alpha} x(0)}{d t^{\alpha}}=x_{1}+h(x)$ mean the nonlocal conditions, which can be applied in physics with better effects than the classical initial conditions [57-59]. We note that the existence result given in [1, Theorem 3.1] for Cauchy problem (1.1) has been proved by using the Krasnoselskii fixed point theorem, under the following assumptions:

$\left(A_{1}\right)$ There exists a constant $L_{1}$ such that $\|g(y)-g(x)\| \leq L_{1}|y-x|_{c}$ for all $y, x \in \mathcal{C}$.

$\left(A_{2}\right)$ There exists a constant $L_{2}$ such that $\|h(y)-h(x)\| \leq L_{2}|y-x|_{c}$ for all $y, x \in \mathcal{C}$.

$\left(A_{3}\right)$ The family $(S(t))_{t \in \mathbb{R}}$ is compact for all $t>0$.

However, there are many concrete applications in which the above assumptions are difficult to realize. Indeed, for $X=L^{2}(\Omega, \mathbb{R})$ with $\Omega$ is a bounded domain in $\mathbb{R}^{n}(n \geq 1)$, many authors [60-62] have considered the following nonlocal condition:

$$
g(x)=\int_{\Omega} \int_{0}^{T} K(t, \cdot, \sigma, x(t)(\sigma)) d t d \sigma
$$

which does not satisfy Lipschitz condition $\left(A_{1}\right)$, where $K$ is a kernel with the following conditions:

$\left(C_{1}\right) K(t, \xi, \sigma, \cdot)$ is a continuous function for almost every $(t, \xi, \sigma) \in[0, T] \times \Omega \times \Omega$.

$\left(C_{2}\right) K(\cdot, \cdot, \cdot, r)$ is a measurable function for each fixed $r \in \mathbb{R}$.

$\left(C_{3}\right)\left|K\left(t, \xi_{1}, \sigma, r\right)-K\left(t, \xi_{2}, \sigma, r\right)\right| \leq m_{k}\left(t, \xi_{1}, \xi_{2}, \sigma\right)$ for all $\left(t, \xi_{1}, \sigma, r\right),\left(t, \xi_{2}, \sigma, r\right) \in$ $[0, T] \times \Omega \times \Omega \times \mathbb{R}$ with $|r| \leq k$, where $m_{k} \in L^{1}\left([0, T] \times \Omega \times \Omega \times \mathbb{R}, \mathbb{R}^{+}\right)$and $\lim _{\xi_{1} \rightarrow \xi_{2}} \int_{\Omega} \int_{0}^{T} m_{k}\left(t, \xi_{1}, \xi_{2}, \sigma\right) d t d \sigma=0$, uniformly in $\xi_{2} \in \Omega$.

(C $\left.C_{4}\right)|K(t, \xi, \sigma, r)| \leq \frac{\delta}{\operatorname{Tm}(\Omega)}|r|+\eta(t, \xi, \sigma)$ for all $r \in \mathbb{R}$ and $\delta>0$, where $\eta \in L^{2}\left([0, T] \times \Omega \times \Omega, \mathbb{R}^{+}\right)$.

Motivated by this discussion, in the present work we use the Darbo-Sadovskii fixed point theorem in order to prove the existence of mild solutions for Cauchy problem (1.1) without assuming the Lipschitz conditions imposed in $\left(A_{1}\right),\left(A_{2}\right)$ and the compactness of the family $(S(t))_{t>0}$.

The rest of this paper is organized as follows. In Sect. 2, we briefly recall some tools related to the conformable fractional calculus, the cosine family of linear operators, and the Hausdorff measure of noncompactness. Section 3 is devoted to proving the main result.

\section{Preliminaries}

We recall some preliminary facts on the conformable fractional calculus. 
Definition 2.1 ([23]) For $\alpha \in] 0,1]$, the conformable fractional derivative of order $\alpha$ of a function $x(\cdot):[0,+\infty[\longrightarrow \mathbb{R}$ is defined as

$$
\frac{d^{\alpha} x(t)}{d t^{\alpha}}=\lim _{\varepsilon \rightarrow 0} \frac{x\left(t+\varepsilon t^{1-\alpha}\right)-x(t)}{\varepsilon} \quad \text { for } t>0 \quad \text { and } \quad \frac{d^{\alpha} x(0)}{d t^{\alpha}}=\lim _{t \rightarrow 0^{+}} \frac{d^{\alpha} x(t)}{d t^{\alpha}}
$$

provided that the limits exist.

The conformable fractional integral $I^{\alpha}(\cdot)$ of a function $x(\cdot)$ is defined by

$$
I^{\alpha}(x)(t)=\int_{0}^{t} s^{\alpha-1} x(s) d s \quad \text { for } t>0 .
$$

Theorem 2.1 ([23]) If $x(\cdot)$ is a continuous function in the domain of $I^{\alpha}(\cdot)$, then we have

$$
\frac{d^{\alpha}\left(I^{\alpha}(x)(t)\right)}{d t^{\alpha}}=x(t)
$$

Theorem $2.2([25])$ If $x(\cdot)$ is a differentiable function, then we have

$$
I^{\alpha}\left(\frac{d^{\alpha} x(\cdot)}{d t^{\alpha}}\right)(t)=x(t)-x(0)
$$

Now, we present some definitions concerning the cosine family of linear operators.

Definition $2.2([55])$ A one parameter family $(C(t))_{t \in \mathbb{R}}$ of bounded linear operators on a Banach space $X$ is called a strongly continuous cosine family if and only if:

1. $C(0)=I$, where $I$ is the identity operator in the space $X$.

2. $C(s+t)+C(s-t)=2 C(s) C(t)$ for all $t, s \in \mathbb{R}$.

3. The function $t \longmapsto C(t) x$ is strongly continuous for each $x \in X$.

We also define the sine family $(S(t))_{t \in \mathbb{R}}$ associated with the cosine family $(C(t))_{t \in \mathbb{R}}$ as follows:

$$
S(t) x=\int_{0}^{t} C(s) x d s, \quad x \in X .
$$

The infinitesimal generator $A$ of a strongly continuous cosine family $((C(t)),(S(t)))_{t \in \mathbb{R}}$ on $X$ is defined by

$$
D(A)=\{x \in X, t \longmapsto C(t) x \text { is a twice continuously differentiable function }\}
$$

and

$$
A x=\frac{d^{2} C(0) x}{d t^{2}}, \quad x \in D(A) .
$$

We end these preliminaries by some concepts on the Hausdorff measure of noncompactness. 
Definition $2.3([63,64])$ The Hausdorff measure of noncompactness $\sigma$ of a bounded set $B$ in a Banach space $X$ is defined as follows:

$$
\sigma(B)=\inf \left\{\varepsilon>0: B=\bigcup_{i=1}^{n} B_{i}, \text { with } \operatorname{diam}\left(B_{i}\right) \leq \varepsilon \text { for } i=1, \ldots, n\right\} .
$$

The following lemma presents some basic properties of the Hausdorff measure of noncompactness.

Lemma 2.1 ([63, 64]) Let $X$ be a Banach space and $B, C \subseteq X$ be bounded sets. Then the following properties hold:

(1) $B$ is pre-compact if and only if $\sigma(B)=0$.

(2) $\sigma(B)=\sigma(\bar{B})=\sigma(\operatorname{conv}(B))$, where $\bar{B}$ and $\operatorname{conv}(B)$ mean the closure and convex hull of $B$, respectively.

(3) $\sigma(B) \leq \sigma(C)$, where $B \subseteq C$.

(4) $\sigma(B+C) \leq \sigma(B)+\sigma(C)$, where $B+C=\{x+y: x \in B, y \in C\}$.

(5) $\sigma(B \cup C) \leq \max \{\sigma(B), \sigma(C)\}$.

(6) $\sigma(\lambda B)=|\lambda| \sigma(B)$ for any $\lambda \in \mathbb{R}$, when $X$ is a real Banach space.

(7) If the operator $Q: D(Q) \subseteq X \rightarrow Y$ is Lipschitz continuous with constant $k$, then we have $\rho(Q(B)) \leq k \sigma(B)$ for any bounded subset $B \subseteq D(Q)$, where $Y$ is another Banach space and $\rho$ represents the Hausdorff measure of noncompactness in $Y$.

Definition 2.4 ([64]) The operator $Q: D(Q) \subseteq X \longrightarrow X$ is said to be an $\sigma$-contraction if there exists a positive constant $k<1$ such that $\sigma(Q(B)) \leq k \sigma(B)$ for any bounded closed subset $B \subseteq D(Q)$.

Lemma 2.2 (Darbo-Sadovskii theorem, [63, 64]) Let $B \subset X$ be a bounded, closed, and convex set. If $Q: B \longrightarrow B$ is a continuous and $\sigma$-contraction operator, then $Q$ has at least one fixed point in $B$.

Lemma $2.3([65,66])$ Let $D \subset X$ be a bounded set, then there exists a countable set $D_{0} \subset D$ such that $\sigma(D) \leq 2 \sigma\left(D_{0}\right)$.

In the sequel, we denote by $\sigma_{c}$ the Hausdorff measure of noncompactness in the space $\mathcal{C}$ of continuous functions $x(\cdot)$ defined from $[0, \tau]$ into $X$ equipped with the norm $|x|_{c}=$ $\sup _{t \in[0, \tau]}\|x(t)\|$. It is well known that the space $\left(\mathcal{C},|\cdot|_{c}\right)$ is a Banach space. $t \in[0, \tau]$

Lemma 2.4 ([67]) Let $D_{0}:=\left\{x_{n}\right\} \subset \mathcal{C}$ be a countable set, then we have

(1) $\sigma\left(D_{0}(t)\right):=\sigma\left(\left\{x_{n}(t)\right\}\right)$ is Lebesgue integrable on $[0, \tau]$.

(2) $\sigma\left(\int_{0}^{\tau} D_{0}(s) d s\right) \leq 2 \int_{0}^{\tau} \sigma\left(D_{0}(s)\right) d s$, where $\sigma\left(\int_{0}^{\tau} D_{0}(s) d s\right):=\sigma\left(\left\{\int_{0}^{\tau} x_{n}(s) d s\right\}\right)$.

Lemma 2.5 ([63]) Let $D \subset \mathcal{C}$ be bounded and equicontinuous, then we have

(1) $\sigma(D(t))$ is continuous on $[0, \tau]$.

(2) $\sigma_{c}(D)=\max _{t \in[0, \tau]}(\sigma(D(t)))$. 


\section{Main result}

According to [1], we have the following definition.

Definition 3.1 A function $x \in \mathcal{C}$ is called a mild solution of Cauchy problem (1.1) if

$$
x(t)=C\left(\frac{t^{\alpha}}{\alpha}\right)\left[x_{0}+g(x)\right]+S\left(\frac{t^{\alpha}}{\alpha}\right)\left[x_{1}+h(x)\right]+\int_{0}^{t} s^{\alpha-1} S\left(\frac{t^{\alpha}-s^{\alpha}}{\alpha}\right) f(s, x(s)) d s .
$$

To obtain the existence of mild solutions, we will need the following assumptions:

$\left(H_{1}\right)$ The function $f(t, \cdot): X \longrightarrow X$ is continuous, and for all $r>0$, there exists a function $\varphi_{r} \in L^{\infty}\left([0, \tau], \mathbb{R}^{+}\right)$such that $\sup \|f(t, x)\| \leq \varphi_{r}(t)$ for all $t \in[0, \tau]$.

$\left(H_{2}\right)$ The function $f(\cdot, x):[0, \tau] \longrightarrow X$ is continuous for all $x \in X$.

$\left(H_{3}\right)$ The function $g: \mathcal{C} \longrightarrow X$ is continuous and compact.

$\left(H_{4}\right)$ The function $h: \mathcal{C} \longrightarrow X$ is continuous and compact.

$\left(H_{5}\right)$ There exist positive constants $a$ and $b$ such that $\|g(x)\| \leq a|x|_{c}+b$ for all $x \in \mathcal{C}$.

$\left(H_{6}\right)$ There exist positive constants $c$ and $d$ such that $\|h(x)\| \leq c|x|_{c}+d$ for all $x \in \mathcal{C}$.

$\left(H_{7}\right)$ There exists a positive constant $L$ such that $\sigma\left(f\left(t, D_{0}\right)\right) \leq L \sigma\left(D_{0}\right)$ for any countable set $D_{0} \subset X$ and $t \in[0, \tau]$.

$\left(H_{8}\right)$ The family $(C(t))_{t \in \mathbb{R}}$ is uniformly continuous, that is, $\lim _{t \rightarrow s}|C(s)-C(t)|=0$, where $|\cdot|$ represents the norm in the space of bounded operators defined from $X$ into itself.

Remark 3.1 The nonlocal condition given in (1.2) satisfies assumption $\left(H_{3}\right)$.

Indeed, the continuity and compactness of the function $g$ are guaranteed by using [68, Theorem 4.2] in view of conditions $\left(C_{1}\right)-\left(C_{4}\right)$ assumed for the kernel $K$. Moreover, by using condition $\left(C_{4}\right)$, we get $a=\frac{\delta}{\sqrt{m(\Omega)}}$ and $b=\sqrt{\operatorname{Tm}(\Omega)}\|\eta\|_{L^{2}\left([0, T] \times \Omega \times \Omega, \mathbb{R}^{+}\right)}$.

Theorem 3.1 Assume that $\left(H_{1}\right)-\left(H_{8}\right)$ hold, then Cauchy problem (1.1) has at least one mild solution provided that

$$
\max \left(a \sup _{t \in[0, \tau]}\left|C\left(\frac{t^{\alpha}}{\alpha}\right)\right|+c \sup _{t \in[0, \tau]}\left|S\left(\frac{t^{\alpha}}{\alpha}\right)\right|, \frac{4 L \tau^{\alpha}}{\alpha} \sup _{t \in[0, \tau]}\left|S\left(\frac{t^{\alpha}}{\alpha}\right)\right|\right)<1
$$

Proof In order to use the Darbo-Sadovskii fixed point theorem, we define the operator $\Gamma: \mathcal{C} \longrightarrow \mathcal{C}$ by

$$
\Gamma(x)(t)=C\left(\frac{t^{\alpha}}{\alpha}\right)\left[x_{0}+g(x)\right]+S\left(\frac{t^{\alpha}}{\alpha}\right)\left[x_{1}+h(x)\right]+\int_{0}^{t} s^{\alpha-1} S\left(\frac{t^{\alpha}-s^{\alpha}}{\alpha}\right) f(s, x(s)) d s .
$$

We also consider the ball $B_{r}:=\left\{x \in \mathcal{C},|x|_{c} \leq r\right\}$, where

$$
r \geq \frac{\sup _{t \in[0, \tau]}\left|C\left(\frac{t^{\alpha}}{\alpha}\right)\right|\left[\left\|x_{0}\right\|+b\right]+\sup _{t \in[0, \tau]}\left|S\left(\frac{t^{\alpha}}{\alpha}\right)\right|\left[\left\|x_{1}\right\|+d+\frac{\tau^{\alpha}}{\alpha}\left|\varphi_{r}\right|_{L^{\infty}\left([0, \tau], \mathbb{R}^{+}\right)}\right]}{1-a \sup _{t \in[0, \tau]}\left|C\left(\frac{t^{\alpha}}{\alpha}\right)\right|-c \sup _{t \in[0, \tau]}\left|S\left(\frac{t^{\alpha}}{\alpha}\right)\right|} .
$$

The proof will be given in four steps.

Step 1: Prove that $\Gamma\left(B_{r}\right) \subset B_{r}$. 
For $x \in \mathcal{C}$, we have

$$
\begin{aligned}
\|\Gamma(x)(t)\| \leq & \left\|C\left(\frac{t^{\alpha}}{\alpha}\right)\left[x_{0}+g(x)\right]\right\|+\left\|S\left(\frac{t^{\alpha}}{\alpha}\right)\left[x_{1}+h(x)\right]\right\| \\
& +\int_{0}^{t} s^{\alpha-1}\left\|S\left(\frac{t^{\alpha}-s^{\alpha}}{\alpha}\right) f(s, x(s))\right\| d s .
\end{aligned}
$$

Using assumptions $\left(H_{1}\right),\left(H_{5}\right)$, and $\left(H_{6}\right)$, we get

$$
\begin{aligned}
\|\Gamma(x)(t)\| \leq & \sup _{t \in[0, \tau]}\left|C\left(\frac{t^{\alpha}}{\alpha}\right)\right|\left[\left\|x_{0}\right\|+a|x|_{c}+b\right] \\
& +\sup _{t \in[0, \tau]}\left|S\left(\frac{t^{\alpha}}{\alpha}\right)\right|\left[\left\|x_{1}\right\|+c|x|_{c}+d+\frac{\tau^{\alpha}}{\alpha}\left|\varphi_{r}\right|_{L^{\infty}\left([0, \tau], \mathbb{R}^{+}\right)}\right] \\
\leq & \sup _{t \in[0, \tau]}\left|C\left(\frac{t^{\alpha}}{\alpha}\right)\right|\left[\left\|x_{0}\right\|+a r+b\right] \\
& +\sup _{t \in[0, \tau]}\left|S\left(\frac{t^{\alpha}}{\alpha}\right)\right|\left[\left\|x_{1}\right\|+c r+d+\frac{\tau^{\alpha}}{\alpha}\left|\varphi_{r}\right|_{L^{\infty}\left([0, \tau], \mathbb{R}^{+}\right)}\right] \\
\leq & r .
\end{aligned}
$$

Taking the supremum, we obtain $|\Gamma(x)|_{c} \leq r$, and this shows that $\Gamma\left(B_{r}\right) \subset B_{r}$.

Step 2: Prove that $\Gamma: B_{r} \longrightarrow B_{r}$ is continuous.

Let $\left(x_{n}\right) \subset B_{r}$ such that $x_{n} \longrightarrow x$ in $B_{r}$. We have

$$
\begin{aligned}
\Gamma\left(x_{n}\right)(t)-\Gamma(x)(t)= & C\left(\frac{t^{\alpha}}{\alpha}\right)\left[g\left(x_{n}\right)-g(x)\right]+S\left(\frac{t^{\alpha}}{\alpha}\right)\left[h\left(x_{n}\right)-h(x)\right] \\
& +\int_{0}^{t} s^{\alpha-1} S\left(\frac{t^{\alpha}-s^{\alpha}}{\alpha}\right)\left[f\left(s, x_{n}(s)\right)-f(s, x(s))\right] d s .
\end{aligned}
$$

Using a simple computation, we obtain

$$
\begin{aligned}
\left|\Gamma\left(x_{n}\right)-\Gamma(x)\right|_{c} \leq & \sup _{t \in[0, \tau]}\left|C\left(\frac{t^{\alpha}}{\alpha}\right)\right|\left\|g\left(x_{n}\right)-g(x)\right\|+\sup _{t \in[0, \tau]}\left|S\left(\frac{t^{\alpha}}{\alpha}\right)\right|\left\|h\left(x_{n}\right)-h(x)\right\| \\
& +\sup _{t \in[0, \tau]}\left|S\left(\frac{t^{\alpha}}{\alpha}\right)\right| \int_{0}^{\tau} s^{\alpha-1}\left\|f\left(s, x_{n}(s)\right)-f(s, x(s))\right\| d s .
\end{aligned}
$$

In view of assumption $\left(H_{1}\right)$, we get $\left\|s^{\alpha-1}\left[f\left(s, x_{n}(s)\right)-f(s, x(s))\right]\right\| \leq 2 s^{\alpha-1} \varphi_{r}(s)$ and $f\left(s, x_{n}(s)\right) \longrightarrow f(s, x(s))$ as $n \longrightarrow+\infty$. Then the Lebesgue dominated convergence theorem proves that

$$
\int_{0}^{\tau} s^{\alpha-1}\left\|f\left(s, x_{n}(s)\right)-f(s, x(s))\right\| d s \longrightarrow 0 \quad \text { as } n \longrightarrow+\infty .
$$

By using the continuity of the functions $g$ and $h$, we deduce that $\lim _{n \rightarrow+\infty}\left\|g\left(x_{n}\right)-g(x)\right\|=0$ and $\lim _{n \rightarrow+\infty}\left\|h\left(x_{n}\right)-h(x)\right\|=0$. Hence the operator $\Gamma$ is continuous.

Step 3: Prove that $\Gamma\left(B_{r}\right)$ is equicontinuous.

For $x \in B_{r}$ and $\mu, \nu \in[0, \tau]$ such that $\mu<\nu$, we have

$$
\Gamma(x)(v)-\Gamma(x)(\mu)=\left[C\left(\frac{v^{\alpha}}{\alpha}\right)-C\left(\frac{\mu^{\alpha}}{\alpha}\right)\right]\left(x_{0}+g(x)\right)
$$


Atraoui and Bouaouid Advances in Difference Equations

(2021) 2021:447

Page 7 of 11

$$
\begin{aligned}
& +\left[S\left(\frac{\nu^{\alpha}}{\alpha}\right)-S\left(\frac{\mu^{\alpha}}{\alpha}\right)\right]\left(x_{1}+h(x)\right) \\
& +\int_{0}^{\mu} s^{\alpha-1}\left[S\left(\frac{\nu^{\alpha}-s^{\alpha}}{\alpha}\right)-S\left(\frac{\mu^{\alpha}-s^{\alpha}}{\alpha}\right)\right] f(s, x(s)) d s \\
& +\int_{\mu}^{v} s^{\alpha-1} S\left(\frac{\nu^{\alpha}-s^{\alpha}}{\alpha}\right) f(s, x(s)) d s .
\end{aligned}
$$

Since $S(t)=\int_{0}^{t} C(\theta) d \theta$, then the above equation can be rewritten as follows:

$$
\begin{aligned}
\Gamma(x)(v)-\Gamma(x)(\mu) & =\left[C\left(\frac{v^{\alpha}}{\alpha}\right)-C\left(\frac{\mu^{\alpha}}{\alpha}\right)\right]\left(x_{0}+g(x)\right)+\left[\int_{\frac{\mu^{\alpha}}{\alpha}}^{\frac{\nu^{\alpha}}{\alpha}} C(\theta)\left(x_{1}+h(x)\right) d \theta\right] \\
& +\int_{0}^{\mu} s^{\alpha-1}\left[\int_{\frac{\mu^{\alpha}-s^{\alpha}}{\alpha}}^{\frac{\nu^{\alpha}-s^{\alpha}}{\alpha}} C(\theta) f(s, x(s)) d \theta\right] d s \\
& +\int_{\mu}^{v} s^{\alpha-1} S\left(\frac{\nu^{\alpha}-s^{\alpha}}{\alpha}\right) f(s, x(s)) d s .
\end{aligned}
$$

Therefore, we obtain

$$
\begin{aligned}
\|\Gamma(x)(v)-\Gamma(x)(\mu)\| \leq & \left|C\left(\frac{\nu^{\alpha}}{\alpha}\right)-C\left(\frac{\mu^{\alpha}}{\alpha}\right)\right|\left\|x_{0}+g(x)\right\| \\
& +\sup _{t \in\left[0, \frac{\tau^{\alpha}}{\alpha}\right]}|C(t)|\left[\int_{\frac{\mu^{\alpha}}{\alpha}}^{\frac{v^{\alpha}}{\alpha}}\left\|x_{1}+h(x)\right\| d \theta\right] \\
& +\sup _{t \in\left[0, \frac{\tau^{\alpha}}{\alpha}\right]}|C(t)| \int_{0}^{\mu} s^{\alpha-1}\left[\int_{\frac{\mu^{\alpha}-s^{\alpha}}{\alpha}}^{\frac{v^{\alpha}-s^{\alpha}}{\alpha}}\|f(s, x(s))\| d \theta\right] d s \\
& +\sup _{t \in[0, \tau]}\left|S\left(\frac{t^{\alpha}}{\alpha}\right)\right| \int_{\mu}^{v} s^{\alpha-1}\|f(s, x(s))\| d s .
\end{aligned}
$$

By using assumptions $\left(H_{1}\right),\left(H_{5}\right)$, and $\left(H_{6}\right)$, we get

$$
\begin{aligned}
\|\Gamma(x)(\nu)-\Gamma(x)(\mu)\| \leq & \left|C\left(\frac{\nu^{\alpha}}{\alpha}\right)-C\left(\frac{\mu^{\alpha}}{\alpha}\right)\right|\left(\left\|x_{0}\right\|+a|x|_{c}+b\right) \\
& +\sup _{t \in\left[0, \frac{\tau^{\alpha}}{\alpha}\right]}|C(t)|\left(\left\|x_{1}\right\|+c|x|_{c}+d\right)\left[\int_{\frac{\mu^{\alpha}}{\alpha}}^{\frac{\nu^{\alpha}}{\alpha}} 1 d \theta\right] \\
& +\sup _{t \in\left[0, \frac{\tau^{\alpha}}{\alpha}\right]}|C(t)|\left|\varphi_{r}\right|_{L^{\infty}\left([0, \tau], \mathbb{R}^{+}\right)}^{\mu} \int_{0}^{\mu} s^{\alpha-1}\left[\int_{\frac{\mu^{\alpha}-s^{\alpha}}{\alpha}}^{\frac{\nu^{\alpha}-s^{\alpha}}{\alpha}} 1 d \theta\right] d s \\
& +\sup _{t \in[0, \tau]}\left|S\left(\frac{t^{\alpha}}{\alpha}\right)\right|\left|\varphi_{r}\right|_{L^{\infty}\left([0, \tau], \mathbb{R}^{+}\right)} \int_{\mu}^{v} s^{\alpha-1} d s .
\end{aligned}
$$

An easy computation now shows that

$$
\begin{aligned}
\|\Gamma(x)(v)-\Gamma(x)(\mu)\| \leq & \left(\left\|x_{0}\right\|+a r+b\right)\left|C\left(\frac{v^{\alpha}}{\alpha}\right)-C\left(\frac{\mu^{\alpha}}{\alpha}\right)\right| \\
& +\sup _{t \in\left[0, \frac{\tau^{\alpha}}{\alpha}\right]}|C(t)|\left(\left\|x_{1}\right\|+c r+d\right)\left[\frac{v^{\alpha}-\mu^{\alpha}}{\alpha}\right]
\end{aligned}
$$




$$
\begin{aligned}
& +\sup _{t \in\left[0, \frac{\tau^{\alpha}}{\alpha}\right]}|C(t)|\left|\varphi_{r}\right|_{L^{\infty}\left([0, \tau], \mathbb{R}^{+}\right)}\left[\frac{\tau^{\alpha}}{\alpha}\right]\left[\frac{\nu^{\alpha}-\mu^{\alpha}}{\alpha}\right] \\
& +\sup _{t \in[0, \tau]}\left|S\left(\frac{t^{\alpha}}{\alpha}\right)\right|\left|\varphi_{r}\right|_{L^{\infty}\left([0, \tau], \mathbb{R}^{+}\right)}\left[\frac{\nu^{\alpha}-\mu^{\alpha}}{\alpha}\right] .
\end{aligned}
$$

The above inequality combined with assumption $\left(H_{8}\right)$ shows that $\Gamma\left(B_{r}\right)$ is equicontinuous on $[0, \tau]$.

Step 4: Prove that $\Gamma: B_{r} \longrightarrow B_{r}$ is a $\sigma_{c}$-contraction operator.

Let $D \subset B_{r}$, then by Lemma 2.3, there exists a countable set $D_{0}$ such that $D_{0}=\left\{x_{n}\right\} \subset$ $D$. Hence, $\Gamma\left(D_{0}\right)$ becomes a countable subset of $\Gamma(D)$. Thus, Lemma 2.3 proves that $\sigma_{c}(\Gamma(D)) \leq 2 \sigma_{c}\left(\Gamma\left(D_{0}\right)\right)$. Since $\Gamma\left(D_{0}\right)$ is bounded and equicontinuous, then by using the second point of Lemma 2.5 , we obtain

$$
\sigma_{c}\left(\Gamma\left(D_{0}\right)\right)=\max _{t \in[0, \tau]}\left(\sigma\left(\Gamma\left(D_{0}\right)(t)\right)\right)
$$

Accordingly, we deduce that

$$
\begin{aligned}
\sigma_{c}(\Gamma(D)) \leq & 2 \sigma_{c}\left(\Gamma\left(D_{0}\right)\right) \\
= & 2 \max _{t \in[0, \tau]}\left(\sigma\left(\Gamma\left(D_{0}\right)(t)\right)\right) \\
= & 2 \max _{t \in[0, \tau]}\left(\sigma \left(C\left(\frac{t^{\alpha}}{\alpha}\right)\left[x_{0}+g\left(D_{0}\right)\right]+S\left(\frac{t^{\alpha}}{\alpha}\right)\left[x_{1}+h\left(D_{0}\right)\right]\right.\right. \\
& \left.\left.+\int_{0}^{t} s^{\alpha-1} S\left(\frac{t^{\alpha}-s^{\alpha}}{\alpha}\right) f\left(s, D_{0}(s)\right) d s\right)\right) .
\end{aligned}
$$

By using point (4) of Lemma 2.1, we get

$$
\begin{aligned}
\sigma_{c}(\Gamma(D)) \leq & 2 \max _{t \in[0, \tau]}\left(\sigma\left(C\left(\frac{t^{\alpha}}{\alpha}\right)\left[x_{0}+g\left(D_{0}\right)\right]\right)\right. \\
& \left.+\sigma\left(S\left(\frac{t^{\alpha}}{\alpha}\right)\left[x_{1}+h\left(D_{0}\right)\right]\right)+\sigma\left(\int_{0}^{t} s^{\alpha-1} S\left(\frac{t^{\alpha}-s^{\alpha}}{\alpha}\right) f\left(s, D_{0}(s)\right) d s\right)\right) .
\end{aligned}
$$

Since $g$ and $h$ are compact, then the sets $C\left(\frac{t^{\alpha}}{\alpha}\right)\left[x_{0}+g\left(D_{0}\right)\right]$ and $S\left(\frac{t^{\alpha}}{\alpha}\right)\left[x_{1}+h\left(D_{0}\right)\right]$ are relatively compact. According to the above equation and the first point of Lemma 2.1, we obtain

$$
\sigma_{c}(\Gamma(D)) \leq 2 \max _{t \in[0, \tau]}\left(\sigma\left(\int_{0}^{t} s^{\alpha-1} S\left(\frac{t^{\alpha}-s^{\alpha}}{\alpha}\right) f\left(s, D_{0}(s)\right) d s\right)\right) .
$$

In view of Lemma 2.4, we get

$$
\sigma_{c}(\Gamma(D)) \leq 4 \max _{t \in[0, \tau]}\left(\int_{0}^{t} s^{\alpha-1} \sigma\left(S\left(\frac{t^{\alpha}-s^{\alpha}}{\alpha}\right) f\left(s, D_{0}(s)\right) d s\right)\right) .
$$

Next, point (7) of Lemma 2.1 shows that

$$
\sigma_{c}(\Gamma(D)) \leq 4 \sup _{t \in[0, \tau]}\left|S\left(\frac{t^{\alpha}}{\alpha}\right)\right| \max _{t \in[0, \tau]}\left(\int_{0}^{t} s^{\alpha-1} \sigma\left(f\left(s, D_{0}(s)\right)\right) d s\right) .
$$


According to assumption $\left(H_{7}\right)$, we get

$$
\sigma_{c}(\Gamma(D)) \leq 4 L \sup _{t \in[0, \tau]}\left(\left|S\left(\frac{t^{\alpha}}{\alpha}\right)\right|\right) \max _{t \in[0, \tau]}\left(\int_{0}^{t} s^{\alpha-1} \sigma\left(D_{0}(s)\right) d s\right) .
$$

Hence, by using a simple computation combined with point (2) of Lemma 2.5, we obtain

$$
\begin{aligned}
\sigma_{c}(\Gamma(D)) & \leq 4 L \sup _{t \in[0, \tau]}\left|S\left(\frac{t^{\alpha}}{\alpha}\right)\right| \sigma_{c}(D) \int_{0}^{\tau} s^{\alpha-1} d s \\
& =4 L \sup _{t \in[0, \tau]}\left|S\left(\frac{t^{\alpha}}{\alpha}\right)\right| \sigma_{c}(D) \frac{\tau^{\alpha}}{\alpha} .
\end{aligned}
$$

Then, we have

$$
\sigma_{c}(\Gamma(D)) \leq \frac{4 L \tau^{\alpha}}{\alpha} \sup _{t \in[0, \tau]}\left|S\left(\frac{t^{\alpha}}{\alpha}\right)\right| \sigma_{c}(D)
$$

Since $\frac{4 L \tau^{\alpha}}{\alpha} \sup _{t \in[0, \tau]}\left|S\left(\frac{t^{\alpha}}{\alpha}\right)\right|<1$, then $\Gamma$ is a $\sigma_{c}$-contraction operator.

In conclusion, Lemma 2.2 shows that the operator $\Gamma$ has at least one fixed point, which is a mild solution of Cauchy problem (1.1).

\section{Conclusion}

Without assuming the Lipschitz condition on the nonlocal conditions and the compactness of the cosine family generated by the linear part, we have proved the existence of mild solutions for a class of nonlocal differential equations of the second order with conformable fractional derivative. The main result is obtained by means of the DarboSadovskii fixed point theorem combined with theory of cosine family of linear operators. The equation studied in the present work can be viewed as an abstract version of the nonlocal conformable fractional telegraph equation considered in the work [31]. As a future work, we will be interested in studying Cauchy problem (1.1) with the non-sequential operator $\frac{d^{2 \alpha} x(t)}{d t^{2 \alpha}}$ instead of the sequential one $\frac{d^{\alpha}}{d t^{\alpha}}\left[\frac{d^{\alpha} x(t)}{d t^{\alpha}}\right]$. This purpose is not easy! Indeed, in this case the cosine family $((C(t)),(S(t)))_{t \in \mathbb{R}}$ must be extended to the complex space $\mathbb{C}$, for more details about this point we refer to the work [1].

Acknowledgements

The authors wish to thank the referees for several valuable and insightful suggestions which improved the quality of this paper. The authors are very grateful to the whole team of the Journal of Advances in Difference Equations for their excellent efforts, and they wish them very great success.

Funding

This research work was not funded by any financial support organization.

Availability of data and materials

No data were used to support this study.

Competing interests

The authors declare that they have no competing interests.

Authors' contributions

The authors declare that the study was realized in collaboration with equal responsibility. All authors read and approved the final manuscript. 


\section{Author details}

1 Department of Mathematics, Faculty of Applied Sciences, Ait Melloul Campus, Ibn Zohr University, BP 6146, 86150, Agadir, Morocco. ${ }^{2}$ Department of Mathematics, Faculty of Sciences and Technics, Sultan Moulay Slimane University, BP 523, 23000, Béni Mellal, Morocco.

\section{Publisher's Note}

Springer Nature remains neutral with regard to jurisdictional claims in published maps and institutional affiliations.

Received: 26 June 2021 Accepted: 12 September 2021 Published online: 10 October 2021

\section{References}

1. Bouaouid, M., Hilal, K., Melliani, S.: Sequential evolution conformable differential equations of second order with nonlocal condition. Adv. Differ. Equ. 2019, 21 (2019)

2. Liu, J.G., Yang, X.J., Feng, Y.Y., Cui, P.: On group analysis of the time fractional extended (2+1)-dimensional Zakharov-Kuznetsov equation in quantum magneto-plasmas. Math. Comput. Simul. 178, 407-421 (2020)

3. Liu, J.G., Yang, X.J., Feng, Y.Y., Cui, P., Geng, L.L.: On integrability of the higher dimensional time fractional KdV-type equation. J. Geom. Phys. 160, 104000 (2021)

4. Liu, J.G., Yang, X.J., Feng, Y.Y., Geng, L.L.: Fundamental results to the weighted Caputo-type differential operator. Appl. Math. Lett. 121, 107421 (2021)

5. Liu, J.G., Yang, X.J., Feng, Y.Y., Iqbal, M.: Group analysis to the time fractional nonlinear wave equation. Int. J. Math. 31, $2050029(2020)$

6. Liu, J.G., Yang, X.J., Feng, Y.Y., Zhang, H.Y.: Analysis of the time fractional nonlinear diffusion equation from diffusion process. J. Appl. Anal. Comput. 10,1060-1072 (2020)

7. Liu, J.G., Yang, X.J., Feng, Y.Y.: Analytical solutions of some integral fractional differential-difference equations. Mod. Phys. Lett. B 34, 2050009 (2020)

8. Liang, X., Gao, F., Zhou, C.B., Wang, Z., Yang, X.J.: An anomalous diffusion model based on a new general fractional operator with the Mittag-Leffler function of Wiman type. Adv. Differ. Equ. 2018, 25 (2018)

9. Phuong, N.D., Hoan, L.V.C., Karapinar, E., Singh, J., Binh, H.D., Can, N.H.: Fractional order continuity of a time semi-linear fractional diffusion-wave system. Alex. Eng. J. 59, 4959-4968 (2020)

10. Phuong, N.D., Tuan, N.A., Kumar, D., Tuan, N.H.: Initial value problem for fractional Volterra integrodifferential pseudo-parabolic equations. Math. Model. Nat. Phenom. 16, 27 (2021)

11. Rashid, S., Chu, Y.M., Singh, J., Kumar, D.: A unifying computational framework for novel estimates involving discrete fractional calculus approaches. Alex. Eng. J. 60, 2677-2685 (2021)

12. Can, N.H., Kumar, D., Vo Viet, T., Nguyen, A.T:: On time fractional pseudo-parabolic equations with non-local in time condition. Math. Methods Appl. Sci. (2021). https://doi.org/10.1002/mma.7196

13. Singh, J., Kumar, D., Baleanu, D.: On the analysis of fractional diabetes model with exponential law. Adv. Differ. Equ. 2018, 231 (2018)

14. Agarwal, R.P., Alsaedi, A., Alghamdi, N., Ntouyas, S.K., Ahmad, B.: Existence results for multi-term fractional differential equations with nonlocal multi-point and multi-strip boundary conditions. Adv. Differ. Equ. 2018, 342 (2018)

15. Jarad, F., Ugurlu, E., Abdeljawad, T., Baleanu, D.: On a new class of fractional operators. Adv. Differ. Equ. 2017, 247 (2017)

16. Samadi, A., Ntouyas, S.K., Tariboon, J.: Nonlocal fractional hybrid boundary value problems involving mixed fractional derivatives and integrals via a generalization of Darbo's theorem. J. Math. 2021, Article ID 6690049 (2021)

17. Sweilam, N.H., Al-Mekhlafi, S.M., Assiri, T., Atangana, A.: Optimal control for cancer treatment mathematical model using Atangana-Baleanu-Caputo fractional derivative. Adv. Differ. Equ. 2020, 334 (2020)

18. Oldham, K.B., Spanier, J.: The Fractional Calculus. Academic Press, San Diego (1974)

19. Miller, K.S., Ross, B.: An Introduction to the Fractional Calculus and Fractional Differential Equations. Wiley, New York (1993)

20. Samko, S.G., Kilbas, A.A., Marichev, O.I.: Fractional Integrals and Derivatives Theory and Applications. Gordon \& Breach Amsterdam (1993)

21. Podlubny, I.: Fractional Differential Equations. Academic Press, San Diego (1999)

22. Kilbas, A.A., Srivastava, H.M., Trujillo, J.J.: Theory and Applications of Fractional Differential Equations. Elsevier, Amesterdam (2006)

23. Khalil, R., Al Horani, M., Yousef, A., Sababheh, M.: A new definition of fractional derivative. J. Comput. Appl. Math. 264, 65-70 (2014)

24. Atangana, A., Baleanu, D.: New fractional derivatives with nonlocal and non-singular kernel: theory and application to heat transfer model. Therm. Sci. (2016). https://doi.org/10.2298/TSCI160111018A

25. Abdeljawad, T.: On conformable fractional calculus. J. Comput. Appl. Math. 279, 57-66 (2015)

26. Chung, W.: Fractional Newton mechanics with conformable fractional derivative. J. Comput. Appl. Math. 290 150-158 (2015)

27. Martínez, L., Rosales, J.J., Carreño, C.A., Lozano, J.M.: Electrical circuits described by fractional conformable derivative. Int. J. Circuit Theory Appl. 46, 1091-1100 (2018)

28. Eslami, M., Rezazadeh, H.: The first integral method for Wu-Zhang system with conformable time-fractional derivative. Calcolo 53, 475-485 (2016)

29. Zhao, D., Luo, M.: General conformable fractional derivative and its physical interpretation. Calcolo 54, $903-917$ (2017)

30. Bouaouid, M., Atraoui, M., Hilal, K., Melliani, S.: Fractional differential equations with nonlocal-delay condition. J. Adv. Math. Stud. 11, 214-225 (2018)

31. Bouaouid, M., Hilal, K., Melliani, S.: Nonlocal telegraph equation in frame of the conformable time-fractional derivative. Adv. Math. Phys. 2019, Article ID 7528937 (2019)

32. Bouaouid, M., Hilal, K., Melliani, S.: Nonlocal conformable fractional Cauchy problem with sectorial operator. Indian J. Pure Appl. Math. 50, 999-1010 (2019) 
33. Bouaouid, M., Hannabou, M., Hilal, K.: Nonlocal conformable-fractional differential equations with a measure of noncompactness in Banach spaces. J. Math. 2020, Article ID 5615080 (2020)

34. Bouaouid, M., Hilal, K., Melliani, S.: Existence of mild solutions for conformable-fractional differential equations with non local conditions. Rocky Mt. J. Math. 50, 871-879 (2020)

35. Bouaouid, M., Hilal, K., Hannabou, M.: Existence and uniqueness of integral solutions to impulsive conformable-fractional differential equations with nonlocal condition. J. Appl. Anal. 27 (2021)

36. Bouaouid, M.: Integral solution of a conformable fractional integro-differential equation with nonlocal condition. Mathematica (2021, to appear)

37. Yang, S., Wang, L., Zhan, S.: Conformable derivative: application to non-Darcian flow in low-permeability porous media. Appl. Math. Lett. 79, 105-110 (2018)

38. Balci, E., Öztürk, I., Kartal, S.: Dynamical behaviour of fractional order tumor model with Caputo and conformable fractional derivative. Chaos Solitons Fractals 123, 43-51 (2019)

39. Tarasov, V.E.: No nonlocality. No fractional derivative. Commun. Nonlinear Sci. Numer. Simul. 62, 157-163 (2018)

40. Al-Refai, M., Abdeljawad, T.: Fundamental results of conformable Sturm-Liouville eigenvalue problems. Complexity 2017, Article ID 3720471 (2017)

41. Lazo, M.J., Torres, D.F.: Variational calculus with conformable fractional derivatives. IEEE/CAA J. Autom. Sin. 4, 340-352 (2017)

42. Ortigueira, M.D., Tenreiro Machado, J.A.: What is a fractional derivative? J. Comput. Phys. 293, 4-13 (2015)

43. Katugampola, U.N.: Correction to "What is a fractional derivative?" by Ortigueira and Machado [Journal of Computational Physics, Volume 293, 15 July 2015, pages 4-13. Special issue on Fractional PDEs]. J. Comput. Phys. 321, 1255-1257 (2016)

44. Abdelhakim, A.A., Tenreiro Machado, J.A.: A critical analysis of the conformable derivative. Nonlinear Dyn. 95, 3063-3073 (2019)

45. Abdelhakim, A.A.: The flaw in the conformable calculus: it is conformable because it is not fractional. Fract. Calc. Appl. Anal. 22, 242-254 (2019)

46. Tuan, N.H., Thach, T.N., Can, N.H., O'Regan, D.: Regularization of a multidimensional diffusion equation with conformable time derivative and discrete data. Math. Methods Appl. Sci. 44, 2879-2891 (2021)

47. Bhanotar, S.A., Kaabar, M.K.: Analytical solutions for the nonlinear partial differential equations using the conformable triple Laplace transform decomposition method. Int. J. Differ. Equ. 2021, Article ID 9988160 (2021)

48. Abbas, M.l.: Existence results and the Ulam stability for fractional differential equations with hybrid proportional-Caputo derivatives. J. Nonlinear Funct. Anal. 2020, Article ID 48 (2020)

49. Abbas, M.l.: Ulam stability and existence results for fractional differential equations with hybrid proportional-Caputo derivatives. J. Interdiscip. Math. (2021). https://doi.org/10.1080/09720502.2021.1889156

50. Gao, F., Chi, C.: Improvement on conformable fractional derivative and its applications in fractional differential equations. J. Funct. Spaces 2020, Article ID 5852414 (2020)

51. Van Au, V., Zhou, Y., Can, N.H., Tuan, N.T.: Regularization of a terminal value nonlinear diffusion equation with conformable time derivative. J. Integral Equ. Appl. 32, 397-416 (2020)

52. Meléndez-Vázquez, F., Fernández-Anaya, G., Hernández-Martínez, E.G.: General conformable estimators with finite-time stability. Adv. Differ. Equ. 2020, 551 (2020)

53. Au, V.V., Baleanu, D., Zhou, Y., Can, N.H.: On a problem for the nonlinear diffusion equation with conformable time derivative. Appl. Anal. 100, 1-25 (2021)

54. Eltayeb, H., Bachar, I., Gad-Allah, M.: Solution of singular one-dimensional Boussinesq equation by using double conformable Laplace decomposition method. Adv. Differ. Equ. 2019, 293 (2019)

55. Travis, C.C., Webb, G.F.: Cosine family and abstract nonlinear second order differential equations. Acta Math. Acad. Sci. Hung. 32, 75-96 (1978)

56. Hernández, E.M.: Existence of solutions to a second order partial differential equation with nonlocal conditions. Electron. J. Differ. Equ. 2003, 51 (2003)

57. Byszewski, L.: Theorems about the existence and uniqueness of solutions of a semilinear evolution nonlocal Cauchy problem. J. Math. Anal. Appl. 162, 494-505 (1991)

58. Deng, K.: Exponential decay of solutions of semilinear parabolic equations with nonlocal initial conditions. J. Math. Anal. Appl. 179, 630-637 (1993)

59. Olmstead, W.E., Roberts, C.A.: The one-dimensional heat equation with a nonlocal initial condition. Appl. Math. Lett. 10, 89-94 (1997)

60. Zhu, L., Li, G.: Existence results of semilinear differential equations with nonlocal initial conditions in Banach spaces. Nonlinear Anal. 74, 5133-5140 (2011)

61. Aizicovici, S., McKibben, M.: Existence results for a class of abstract nonlocal Cauchy problems. Nonlinear Anal. 39 649-668 (2000)

62. Xue, X.: Nonlocal nonlinear differential equations with a measure of noncompactness in Banach spaces. Nonlinear Anal. 70, 2593-2601 (2009)

63. Banas̀, J., Goebel, K.: Measures of Noncompactness in Banach Spaces. Lecture Notes in Pure and Applied Mathematics. Dekker, New York (1980)

64. Deimling, K.: Nonlinear Functional Analysis. Springer, New York (1985)

65. Chen, P., Li, Y.: Monotone iterative technique for a class of semilinear evolution equations with nonlocal conditions. Results Math. 63, 731-744 (2013)

66. Li, Y.: Existence of solutions of initial value problems for abstract semilinear evolution equations. Acta Math. Sinica (Chin. Ser.) 48, 1089-1094 (2005)

67. Heinz, H.P.: On the behaviour of measure of noncompactness with respect to differentiation and integration of vector-valued functions. Nonlinear Anal. 7, 1351-1371 (1983)

68. Martin, R.H.: Nonlinear Operators and Differential Equations in Banach Spaces. Wiley, New York (1976) 